\title{
Wearable Haptics for Remote Social Walking
}

\author{
Tommaso Lisini Baldi ${ }^{1}$,Gianluca Paolocci ${ }^{1,2}$,Davide Barcelli ${ }^{1}$, and Domenico Prattichizzo ${ }^{1,2}$
}

\begin{abstract}
Walking is an essential activity for a healthy life, which becomes less tiring and more enjoyable if done together. Common difficulties we have in performing sufficient physical exercise, for instance the lack of motivation, can be overcome by exploiting its social aspect. However, our lifestyle sometimes makes it very difficult to find time together with others who live far away from us to go for a walk. In this paper we propose a novel system enabling people to have a 'remote social walk' by streaming the gait cadence between two persons walking in different places, increasing the sense of mutual presence. Vibrations provided at the users' ankles display the partner's sensation perceived during the heel-strike. In order to achieve the aforementioned goal in a two users experiment, we envisaged a four-step incremental validation process: $i$ ) a single walker has to adapt the cadence with a virtual reference generated by a software; ii) a single user is tasked to follow a predefined timevarying gait cadence; iii) a leader-follower scenario in which the haptic actuation is mono-directional; $i v$ ) a peer-to-peer case with bi-directional haptic communication. Careful experimental validation was conducted involving a total of 50 participants, which confirmed the efficacy of our system in perceiving the partners' gait cadence in each of the proposed scenarios.
\end{abstract}

Index Terms-Haptics, Wearable Haptics, Telepresence, Social Walking, Cadence Alignment.

\section{INTRODUCTION}

Clapping hands in an audience, playing music in an orchestra, training in sports and dance represent a tiny fraction among the countless situations in which humans perform coordinate actions. Coordinated motion is probably one of the most ancient and exploited human behaviors. For instance, religions around the world incorporate synchronous singing and gestures into their rituals. Psychologists, anthropologists, and sociologists have speculated that rituals involving synchronous moves may produce positive emotions that encourage participation. Wiltermuth and Heat in [1] studied whether synchronous activities serve as a partial solution to the freerider problem facing groups that need to motivate their members to contribute toward the collective good. The physical synchronization mechanism, which occurs when people move in time with one another, has been studied and discussed for decades. In [2], [3], and [4] authors demonstrate that dance can also weaken the boundaries between the self and the group.

The motivation generated by being part of a group can encourage healthy behaviors, as in the 'Social Walking' [5]. Studies on its impact showed an increase in positive attitude

\footnotetext{
${ }^{1}$ Tommaso Lisini Baldi, Gianluca Paolocci, Davide Barcelli, and Domenico Prattichizzo are with the Department of Information Engineering and Mathematics, University of Siena, Via Roma 56, I-53100 Siena, Italy. \{lisini, paolocci, barcelli, prattichizzo\}@diism.unisi.it

${ }^{2}$ Gianluca Paolocci and Domenico Prattichizzo are with the Department of Advanced Robotics, Istituto Italiano di Tecnologia, Genova, 16163, Italy \{gianluca.paolocci, domenico.prattichizzo\}@iit.it
}

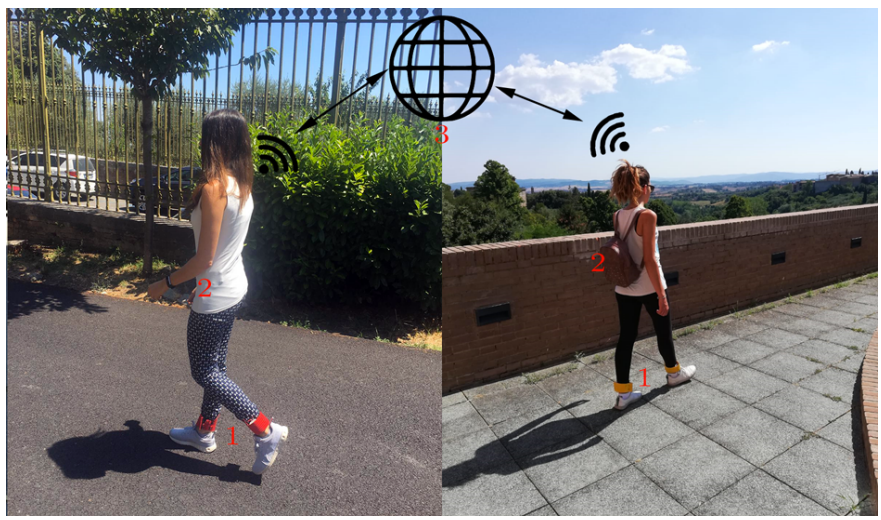

Fig. 1: The proposed remote social walking system: $i$ ) the anklet device (1) measures user $A$ gait cadence; $i i)$ the smartphone (2) receives the measurement via Bluetooth; iii) the information is sent to a server via the Internet $(3)$; $i v)$ the server sends the update to the user B's smart-phone; $v$ ) the smart-phone (2) commands vibrations at the user's anklets (1).

toward physical activity, social cohesion, as well as reducing disease risks [6]. In fact, it has been demonstrated that walking experiences contribute to participants' well-being [7]. Therefore, its promotion as part of the lifestyle has become one of the main aims of governments, as it is expected to significantly reduce health care costs and increase the quality of life [8].

Not many years ago, walking was the primary mean of transport people used to get to work. Nowadays, the great majority of industrialized countries population use cars or public transports to move within the city for daily activities [9]. It follows that walking for a sufficient distance on a weekly basis must be incorporated in the spare time. A recurrent cause of physical inactivity is the lack of time, or often, an appropriate motivation. Although participating in group activities proved a powerful mean to fight physical inactivity, finding the right walking partner may be difficult. In fact, to have a social walk the group needs to share the same time slots and, obvious but essential, the common location where the tour takes place, e.g. the city park. Connecting people far in space may solve the problem. With this work, we aim at creating a system for "feeling" a partner remotely while walking, by exploiting the internet connection to exchange vibrotactile cues, as sketched in Fig. 1 Towards the same goal, Muller et al. presented a headset to transmit gait cadence information through audio cues [10]. Audio signals are often exploited to suggest body postures. For instance, in [11] the authors investigated agency for the entire body by testing auditory action effects related to gait, while Murata et al. proposed a system that synchronizes each step with the music being 
listened to and creates a feeling of generating music through walking [12]. Those systems, however, occupy the hearing, one of the most used input channels.

In our experience the audio channel is a primary source of information about the surroundings, thus cadence cues should be conveyed using different modalities. Haptic communication is generally the preferred option, due to its intuitiveness, efficacy, and intimate nature. Following these motivations, we decided to display cadence information through the use of touch. Tactile feedback has been demonstrated to be an effective way to significantly improve the perceived virtual and social presence of a remote companion. In [13], authors show that haptic feedback reinforces the impression that one is actually there in a mediated environment.

Tactile perceptions can be rendered using haptic interfaces, which apply different kinds of stimuli to the human body that are easily associated with realistic sensations. Haptic interfaces can either rely on kinaesthetic or cutaneous interaction [14]. The former consists in the proprioception of ligaments and muscles tension, necessary for the awareness of limb posture and to estimate external forces. The cutaneous counterpart relies on skin receptors to perceive details like texture, temperature, and shape. Kinaesthetic actuators are not suitable for the proposed work since they are mostly grounded or bulky, while cutaneous stimuli can be displayed by means of small and wearable devices, making them the most appropriate choice for the purpose.

The main categories of wearable cutaneous devices are: skin indentation, skin stretch, temperature, and vibrations [15]. Skin indentation and stretch devices usually can exert forces (normal or tangential, respectively), while vibrations can be modulated to display textures or can be used to render events.

Over the years, cutaneous stimuli have been found an effective, yet non-intrusive, way for suggesting directions and pace cues to users. A vibrotactile waist belt composed of eight tactors was used for waypoint navigation in outdoor scenarios [16]. The waist belt displayed both the direction and distance to the next waypoint. A similar device was used to provide vibrotactile cues for improving situational awareness of soldiers in a simulated building-clearing exercise [17]. In [18], a vibrotactile belt was used for human guidance in indoor environments. Continuous stimuli were used to display directional and rotational motions to the blindfolded users. Vibrotactile armbands were used to navigate subjects along fixed paths using three different stimuli [19]. Similar devices and strategies were used to guide blindfolded users in dynamic environments autonomously [20] or assisted by a mobile robot [21].

For what concerns suggesting the step cadence, exploratory research in this direction revealed the potentiality of using haptics for suggesting rhythm. For instance, in [22] and [23] authors exploited vibrations as a metronome for suggesting tempo in walking/running activities.

With the objective of removing the spatial constraint and establishing a remote presence, we propose a system that measures the gait cadence of each participant and transmits it to the partner, allowing each walker to 'feel' the other. The system, is composed of vibrotactile devices worn at the ankles which provide timing cues displaying the partner's walking pace. The gait cadence is measured using a pressure sensor immersed into a silicon heel insole and connected with one of the haptic interfaces. The detected steps are then sent to the user's smart-phone that communicates with a dedicated server. Finally, each social walker's smart-phone receives the gait cadence update from the server and adjusts the vibration pattern consequently. To the best of our knowledge, this work represents one of the first attempts to allow user dyads to walk together, despite their distance.

This paper is organized as follows. Sect. II provides a detailed description of the proposed system from an engineering perspective, including hardware (haptic anklets) and software (firmware, application and server) details. The third section (Sect. III) presents a divide et impera approach to the problem. We identified four objectives of incremental difficulty for remote social walking to succeed. Sect. IV describes the experiments performed to verify the achievement of these objectives and reports a-posteriori discussions, enriched with correlational analysis of participants' baseline walking parameters and performance in Sect. V] In Sect. VI, qualitative results and users' feedback are reported. Conclusions are drawn in Sect. VII, along with a brief discussion on the range of possible reach directions that the developed system may enable.

\section{SYSTEM OVERVIEW}

The proposed system is composed of three elements: $i$ ) an anklets pair: two wearable fabric-made bands equipped with an electronic board; $i$ ) an application running on a smartphone; iii) a remote server for broadcasting and logging data. During the remote social walk each user has an anklets pair and a smart-phone connected to the server.

\section{A. Description of the anklet device}

The purpose of the wearable devices is twofold: providing the user with vibrotactile stimuli and extracting the gait cadence. The two devices, worn on the two ankles, equip an electronic board with a Bluetooth module and two vibromotors. The devices in a pair differ by the presence of a pressure sensor for cadence sampling. From here on, the sensing anklet will be referred to as master.

Tactile vibratory sensitivity is influenced by the physical location of tactile receptors on the body, their distance with respect to the actuators, the stimulation frequency, and the age of the user. Studies demonstrated that vibrations are better sensed on hairy skin due to its thickness and nerve depth, and that vibrotactile stimuli are best detected in bony areas [24]. In particular, wrists and spine are generally preferred for detecting vibrations, with arms and ankles next in line [25]. A previous study showed that during walking activities participants preferred being informed with haptic cues delivered at the ankles [26]. Due to the aforementioned considerations and since our aim is to design an intuitive and non-obtrusive device which could be easily worn, we concentrated on the development of vibrotactile anklets. Starting from the results 
presented by Scheggi et al. in [27], we decided to use the bilateral configuration, that required two vibro-tactile interfaces, one per ankle.

From a technical point of view, the vibrotactile anklets are composed by cylindrical vibro-motors, independently controlled via Bluetooth with a custom communication protocol (see Fig. 2). The communication between the haptic interface and the smart-phone is realized with an RN-42 Bluetooth antenna connected to the serial port of a $3.3 \mathrm{~V}$ Arduino pro-mini. The wireless connection baud rate is $57600 \mathrm{bps}$. The microcontroller installed on the board is used to pilot the motors activation and to receive data from an external source. Note that each vibro-motor is voltage controlled, which implies a strict coupling between frequency and amplitude that cannot be varied independently. As the user's maximal sensitivity is achieved around $200-300 \mathrm{~Hz}$ [28] (the human sensitive range is between $20 \mathrm{~Hz}$ and $400 \mathrm{~Hz}$ ), two Precision Microdrives Pico Vibe vibration motors are placed into two fabric pockets (the width of the fabric band is about $60 \mathrm{~mm}$ ), with vertically aligned shafts. The motors have a vibration frequency range of $100-300 \mathrm{~Hz}$, lag time of about $20 \mathrm{~ms}$, rise and stop time of $35 \mathrm{~ms}$. The bracelet guarantees about 4 hours of battery life with one motor permanently activated. Each bracelet weights about $90 \mathrm{~g}$.

The micro-controller permanently checks for incoming data on the serial port and, upon reception, activates the motors for $150 \mathrm{~ms}$. This design choice allows for a finer control on the vibrational cues timing at the smart-phone application level, and proved to be the most effective strategy for a prompter adaptation to new gait cadences during the system development. In addition to the previously described hardware, the master anklet is equipped with a flexible force sensor (FSR 400, manufactured by Interlink Electronics, Inc.) connected to the controller. During experimental trials, the force sensor is placed inside the right shoe (under the heel) to record the force pattern due to the contact between foot and ground. The force sensing resistor measures the force applied through the deformation of the active surface, which produces a resistance variation. The force value is converted into a 10 bit digital signal. The stride extraction procedure exploits a single-threshold value, defined as the double of the standard deviation of the data, measured during a calibration phase. The sensor records the pressure under the heel at $100 \mathrm{~Hz}$. Thus, we are able to extract the stride temporal sequence from the obtained data. The stride-detection procedure consists of three phases. In the first step, raw force data are acquired by the system, normalized, and transformed into a two-levels signal using the computed threshold. A square wave is generated as follows: the signal assumes logical value 1 whenever the foot is in contact with the ground, and 0 otherwise. Before further processing, a debounce software mechanism is adopted to rejects variations that are physically improbable. Then, the algorithm extracts positive edges representing the heel contact with the floor, identifying the current stride duration as the time interval between two consecutive edges. When a cadence variation is detected, the new value is sent over the serial port so that the Bluetooth module can deliver it to the paired smart-phone. Moreover, upon establishing a solid

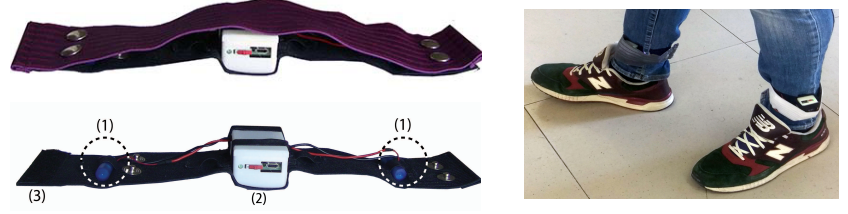

Fig. 2: Haptic cues are provided to the users via two vibrotactile interfaces placed on the ankles. The interfaces are composed of two vibrating motors (1) attached to an elastic band (3). A Li-Ion battery and an Arduino board are in (2).

proof of concept and for a wider experimental validation, it would be possible to move to a more robust, small and easy-to-use device to estimate the user's gait cadence. In that respect, we investigated the usage of one of the most famous sport gadgets: the Nike+iPod Sport Kit (Apple Computer, Inc.) [29]. This solution removes the wear and tear of the pressure sensor and increases the battery lifetime. Details, interfacing, and recording strategies are reported at the end of this manuscript. However, we opted to use the pressure sensor for the present work, because the Nike+iPod Sport Kit guarantees an inferior temporal resolution and introduces additional delay. Such inconveniences would have a negligible impact on a larger scale session as they would be canceled out in the long run.

\section{B. Smart phone app and remote server}

The experimental evaluations presented in this paper leveraged on different pieces of software whose purpose was to regulate the haptic stimulation in real time and to record the gait data during trials, even in an unstructured environment. The overall software architecture employed in this work is organized as follows.

A Java TCP server, hosted in our university facility, permanently accepts incoming connections on a dedicated pair of public IP address and port, while the smart-phones use their cellular network to connect to it over the Internet. The server handles each connection in a dedicated thread, so as to ensure scalability. When the desired number of walkers have established a connection (without loss of generality, in this paper we study the case of two) the server notifies all actors and the information stream is started: whenever a client sends an update the server broadcasts it to all the other clients. In correspondence to any such event, the server also logs the entire state in a text file, for later post-processing. On the smart-phone side, an application is organized with background services to handle both connections, i.e. TCP toward the remote server and Bluetooth toward the Arduino boards. Two main cases are envisaged: $i$ ) a gait update is received from an anklet, which implies an immediate transmission of that information to the server and, in turns, to the other smartphone; ii) an update is received from the network and the local vibration frequency needs to be adjusted accordingly.

Vibrations are managed by the application, depicted in Fig. 3, which employs timers to send the start vibration command to the Arduino boards. Clearly, the anklets vibrations should exhibit a 180 degrees phase, thus the app sends the 


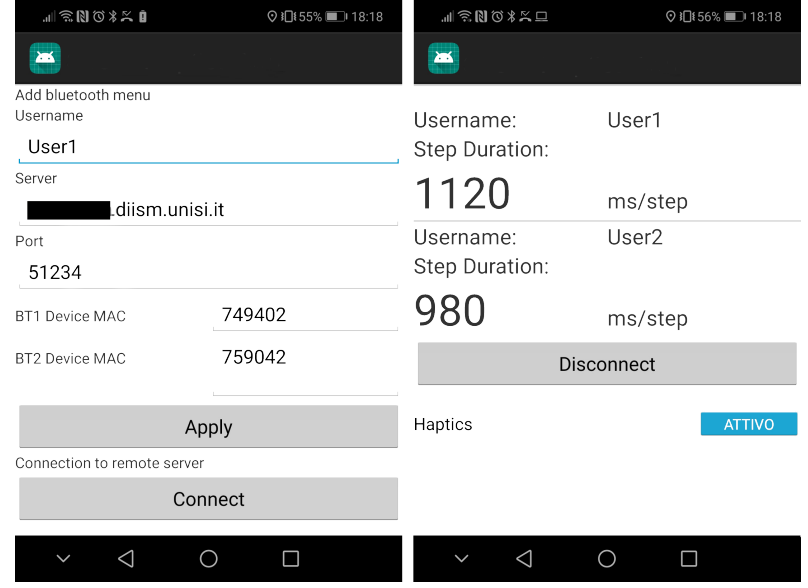

Fig. 3: The smart-phone application used to connect to the remote server via internet and to the anklet via Bluetooth. In the left panel is the initial view of the app. The user can select the username and pair the two haptic anklets using the MAC address. The right panel shows the app screen during a trial. User's and companion's stride duration are visualized by the app. Moreover, a button allows the user to enable or disable the haptic cues manually.

vibration signal alternately to one side or the other, then waits half a period before the next vibration. This design allows for a quick gait cadence adaptation as only half period has to be waited to adjust the vibration timings, while also retaining operation smoothness. The correctness, effectiveness and reliability aspects of the system were analyzed carefully, however we omit the description of the validation process due to space requirements. It is still meaningful though to mention a few considerations in this regard. The overall time needed to observe a vibration frequency update commanded by the other smart-phone is negligible with respect to the system time scales; in fact the $4 \mathrm{G}$ internet connection on the smart-phones introduced almost no delay and the dedicated faculty server was endowed with more than enough bandwidth and tiny ping timing. With regard to data protection, threats must be evaluated since user's data flow over untrustworthy networks. In our case, no sensible information was contained in the packets, which, in addition to the fact that the server port was not previously used and the server was turned on only during experiments, reduced the probability of data corruption and/or stealing.

\section{Human WALKING PACE REgUlation}

In this work, we rely on the gait cycle schema proposed by Philippson in [30]. A stride is completed after a stance and swing phase of a given foot, from heel strike to heel strike. Walking kinematics are characterized by pseudo-periodic patterns, where the stride represents the single period. The temporal duration of a stride is called gait cycle or stride duration, and the distance covered by two consecutive heel strikes of the same foot is called stride length. The application framework of our study is the remote connection between two or more users, thus we cannot provide intuitive indications to regulate the stride length. Instead, we investigate the temporal properties of human gait, i.e. human gait cadence, without considering stride length and walking speed. Notice that we will talk about walking cadence and stride duration referring to the same concept.

The temporal aspects of gait are regulated by (internal) human timekeeping mechanisms and environmental parameters. The synchronization of brain oscillations (and thus motor coordination) with external stimuli relies on a feature of human sensory-motor system called sensory-motor entrainment [31], [32]. If a subject is given a periodic stimulus of constant frequency and sufficient intensity to excite the thalamus, the brain has the tendency to align its dominant EEG frequency to the frequency of the external stimulus. Entrainment applies for visual, audio and haptic stimuli [33]. In our method, participants are periodically provided with vibro-tactile stimuli to suggest the appropriate gait cadence. We positioned the cueing system following results presented in [26]. Generally, the human locomotor system incorporates inputs from both the central nervous system, peripheral inputs, and sensory feedbacks. As reported in [34], these kinds of inputs are suggested to be possible reasons for the presence of the long range correlation in normal human walking. Thus, a gait cadence synchronization method based on body tactile stimulation is suitable for comfortably and effectively suggesting walking cadence.

To test the effectiveness of our system and study the mutual effect of tactile stimuli on users' gait cadence, we introduced a set of 'incremental' experiments, each tailored to assess a specific goal. The rest of this section is devoted to the introduction of the hypotheses motivating each experiment, to facilitate the reading and comprehension of the experimental procedure.

\section{A. Preparatory experiments}

As a preparatory phase, we conducted an experimental campaign to assess whether humans are able to synchronize gait events, e.g. the heel strike or lift off, with vibrations displaying a constant rhythm close to the participant's walking cadence.

Two hypotheses were formulated and tested: $i$ ) humans are capable of aligning their stride sequence to an external rhythm displayed using vibrations; $i i$ ) humans can maintain the suggested walking cadence in presence of a simple secondary task. While the first hypothesis lays the basis for future experiments, the second is tested to understand if it is possible to perform actions which do not require heavy cognitive load while using our system.

\section{B. Artificial constant reference}

As a second phase, we conducted the analysis of human synchronization with an external haptic stimulation by investigating the dependence of human alignment performance from the sign of the cadence variation. In this experiment, participants were tasked to match their stride frequency with paces both slower and faster than the baseline. 


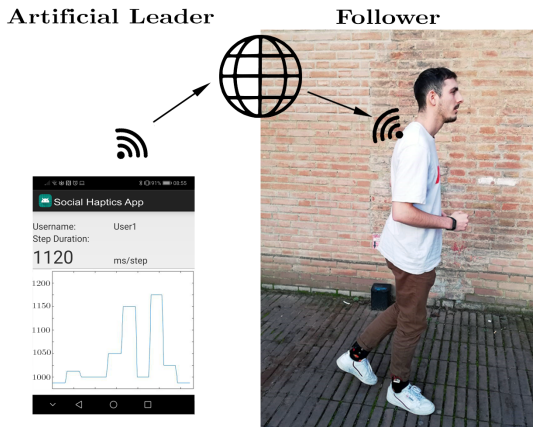

(a) Artificial Leader

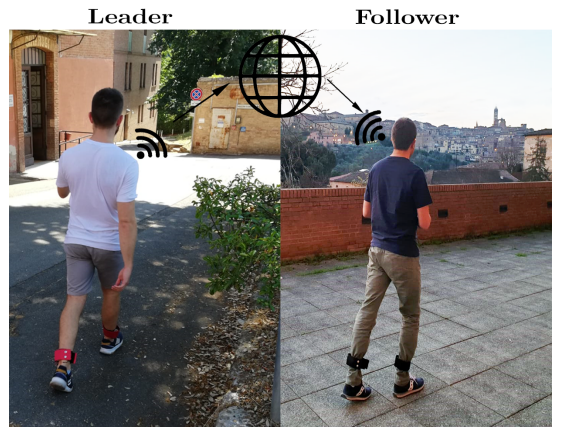

(b) Human Leader

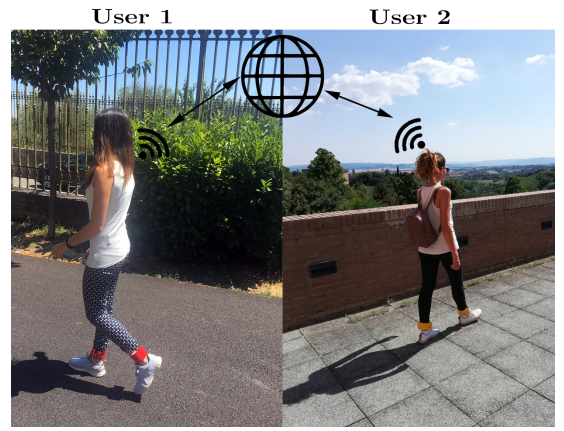

(c) Peer-to-Peer

Fig. 4: In (a) we examined the human capability in entraining to an external rhythm generated by an algorithm. The suggested cadence could be either constant, as in the Artificial Constant Reference experiment (Sect. III-B), or time-varying (Sect. [III-C). In (b) a representative frame of the "Human Leader" experiment (Sect. III-D) is depicted. The follower is asked to synchronize to the gait pace of another human using the proposed system. This experiment differs from the previous one since the human's stride duration is not regular, but features small unpredictable variations. Finally, in the "Peer-to-peer" configuration (c) we tested the bidirectional capability of our system (see Sect. III-E). Both participants were sending each other their respective gait cadence (sensed by their anklet devices). The direction of the information is graphically represented by the arrows.

\section{Artificial leader}

As a further step towards the goal of creating a remote walking system, we had to verify whether humans promptly respond to frequency variations of the haptic cues.

We designed the simplest situation for measuring to which extent a human is capable of adjusting his gait cadence to match a time-varying reference (Fig. 4a). Each trial involved only one user who was instructed to follow as close as possible an external rhythm. The stimulus frequency was updated every 30 seconds while remaining in a specific interval. The purpose of this experiment is to determine whether a properly instructed human can adapt with ease to a timevarying cadence. Therefore we measured the discrepancies between the reference and the human cadence.

\section{Human leader}

The next step toward the remote social walking was replacing the computer program adopted in the 'Artificial Leader' experiment with an actual human. The purpose of this scientific question was to determine whether a human can follow the gait cadence of another human using the proposed system (Fig. 4b). This experiment differs from the previous one since the human walking pace is not as regular as an artificially generated rhythm, and can have small unpredictable variations. It is essential to notice that in this scenario the communication was restricted to one direction only: gait cadence values measured on the leader side were sent over to the follower, while no action was taken upon follower cadence variations. This asymmetry was designed to nullify any synchronization dynamic.

The human follower was instructed to adapt to the perceived gait cadence, whereas the human leader was allowed to walk at his own pace. The leader received no notification regarding the presence of the follower, thus he/she was fully unaware of whether the follower was feeling his/her steps. We measured discrepancies between leader's and follower's gait cadences to study the time needed for the follower to adjust his/her pace and the stability level he/she was capable of maintaining.

\section{E. Peer-to-peer}

In an in-person social walk, i.e. when a group of at least two humans walk together, it is an implicit rule that of adjusting the speed so that the group can clump and stick together. Therefore, in the general case there are no specific roles to be given, such as leader and follower, and each participant would contribute to reach an agreed advancement pace.

This is the scenario in which the full capability of our system emerges: both participants were sending their respective gait cadence (sensed by their anklet devices) to the server which broadcasted them in real time to the partner's smartphone that, in turns, applied the vibration frequency (Fig. 4c). It follows that if the two walkers advanced with different cadences they would have both experienced a misalignment between their steps and the anklets vibrations.

The experimental guidelines in this case play a paramount role as the feedback loop is established and several consensus dynamics are possible: the instructions determine which of those effectively takes place. We decided to instruct the participants to pursue two competitive goals simultaneously and with the same priority: try to adapt to the other's gait cadence but also try to maintain a comfortable walking, as close as possible to one's own natural cadence.

The scientific questions relevant to this experiment are more articulated than the previous cases: while it is still worth studying the transient before stabilizing to a common cadence, it is also interesting to determine the absolute and relative discrepancies between the common and personal cadences. Moreover, comparing the agreed cadence with the average of the two personal ones would be indicative in terms of the consensus dynamics between the participants. 


\begin{tabular}{|c|c|c|c|c|c|c|c|}
\hline \multirow[t]{2}{*}{ Name } & \multicolumn{3}{|c|}{ Haptics } & \multicolumn{3}{|c|}{ Haptics + Secondary Task } & \multirow[t]{2}{*}{ Note } \\
\hline & $2 \%$ & $4 \%$ & $6 \%$ & $2 \%$ & $4 \%$ & $6 \%$ & \\
\hline User1 & 66,32 & 99,05 & 100,00 & 47,36 & 84,88 & 92,59 & \\
\hline User2 & 89,06 & 100,00 & 100,00 & 88,19 & 100,00 & 100,00 & $\star$ \\
\hline User3 & 78,90 & 98,00 & 99,80 & 28,85 & 78,19 & 99,60 & \\
\hline User4 & 47,35 & 98,83 & 100,00 & 78,66 & 100,00 & 100,00 & \\
\hline User5 & 91,13 & 100,00 & 100,00 & 38,88 & 77,34 & 87,43 & \\
\hline User6 & 81,52 & 96,53 & 100,00 & 55,82 & 93,43 & 100,00 & \\
\hline User7 & 89,95 & 99,69 & 100,00 & 36,91 & 86,95 & 100,00 & \\
\hline User8 & 94,78 & 100,00 & 100,00 & 25,19 & 78,78 & 100,00 & \\
\hline User9 & 76,98 & 98,47 & 99,96 & 44,80 & 80,38 & 92,29 & \\
\hline User10 & 80,38 & 100,00 & 100,00 & 67,61 & 100,00 & 100,00 & $\star$ \\
\hline User11 & 66,58 & 94,41 & 100,00 & 0,00 & 4,20 & 39,82 & \\
\hline User12 & 41,50 & 94,30 & 99,52 & 34,45 & 98,54 & 100,00 & \\
\hline User13 & 83,28 & 100,00 & 100,00 & 48,96 & 97,32 & 100,00 & \\
\hline User14 & 78,77 & 94,68 & 100,00 & 19,07 & 62,93 & 97,33 & \\
\hline User15 & 76,01 & 99,50 & 100,00 & 76,01 & 99,52 & 100,00 & \\
\hline User16 & 90,76 & 100,00 & 100,00 & 47,63 & 84,67 & 94,27 & \\
\hline User17 & 77,10 & 98,56 & 100,00 & 49,64 & 81,36 & 93,68 & \\
\hline User18 & 88,35 & 99,60 & 100,00 & 50,88 & 92,36 & 100,00 & \\
\hline User19 & 73,78 & 88,66 & 99,09 & 53,58 & 88,04 & 91,48 & \\
\hline User20 & 72,27 & 100,00 & 100,00 & 39,28 & 82,75 & 95,68 & \\
\hline Percentile 25 & 72.65 & 96.90 & 100.00 & 35.07 & 79.18 & 92.86 & \\
\hline Percentile 50 & 78.84 & 99.28 & 100.00 & 47.50 & 85.92 & 99.80 & \\
\hline Percentile 75 & 88.88 & 100.00 & 100.00 & 55.26 & 98.24 & 100.00 & \\
\hline
\end{tabular}

TABLE I: For each user are reported the percentages of trial duration during which the participant aligned his cadence with the reference rhythm, grouped wrt the considered error bands. Please notice that users tagged with $\star$ are the two high level music players.

\section{EXPERIMENTAL VALIDATION}

As previously mentioned in the introduction (Sect. I) we conducted a step-wise validation. In this Section, we retrace the progression of the experimental process describing experimental protocol, setup, and results per each step.

All the experiments have been held at the open-air athletics track in Siena. Participants were provided with written informed consent and suggested to wear sport equipment. The experimental campaign was held in 5 non-consecutive days, one per experiment, and subjects could discontinue participation at any time. Some participants took part in multiple experimental sessions. All were healthy and none had neuromuscolar disorders or recent injuries at the time of study. It is important to point out that in all the trials involving two participants, they were instructed to walk along different paths, avoiding any visual and audio interaction. The only way of communicating was through haptic stimuli.

\section{A. Preparatory experiments}

In this experiment we evaluated the human capability in adapting the gait cadence to an external constant rhythm. Moreover, we tested whether the addition of a secondary task did affect the cadence synchronization performance.

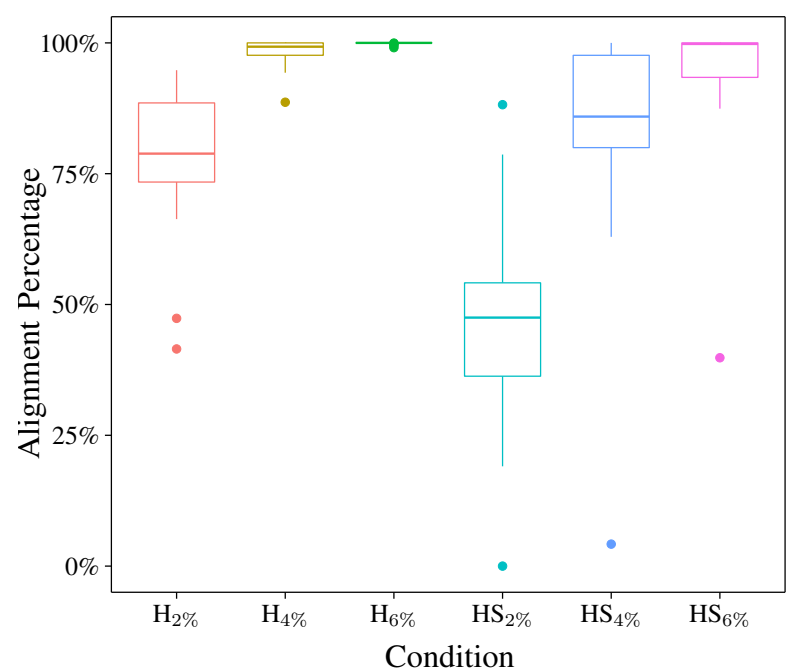

Fig. 5: Boxplots represent the distributions of alignment percentages for each condition. Labels $\mathbf{H}$ and $\mathbf{H S}$ refer to data acquired during trials with haptic stimulation and haptic stimulation and secondary task, respectively. The footnote describes the tolerance band used to discriminate aligned strides from misaligned. The percentage is referred to each subject's baseline stride duration.

The representative sample consisted of twenty subjects (age $31.7 \pm 10.6$ ) with these characteristics: 10 females and 10 males; 6 had previous experiences with haptic interfaces; 2 played music instruments at high level (drums and piano); 12 played sport, two of them in a professional league with regular training sessions.

Participants were provided with an Android phone and two haptic interfaces. The pressure sensor, connected to the master anklet, was positioned under the right heel to sample pressure data and extract stride durations, which were then transmitted via Bluetooth to the smart-phone and logged by the server. In all the trials, participants walked while wearing headphones reproducing white noise to avoid entrainment due to the sound of vibrations and external stimuli.

The first experiment was performed to test the first hypothesis: "can humans synchronize their gait cadence with the rhythm suggested by the anklets?" For each participant the experimental session started with a preliminary acquisition of self-paced gait along a $200 \mathrm{~m}$ path, to record the user's comfortable cadence. In the second trial the haptic interfaces were activated at a frequency $10 \%$ faster than the previously estimated baseline stride duration. Subjects were instructed to align their step sequence to the vibrations during the $200 \mathrm{~m}$ walk.

The second hypothesis was tested by adding a secondary task to the experimental protocol described in the first experiment. The purpose of the secondary task was to determine whether the presence of additional mental efforts affected the users' ability to follow the rhythm dictated by the haptics. 


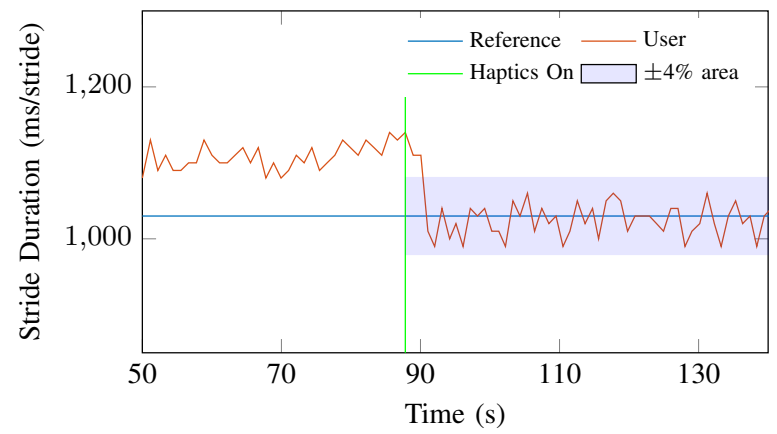

(a)

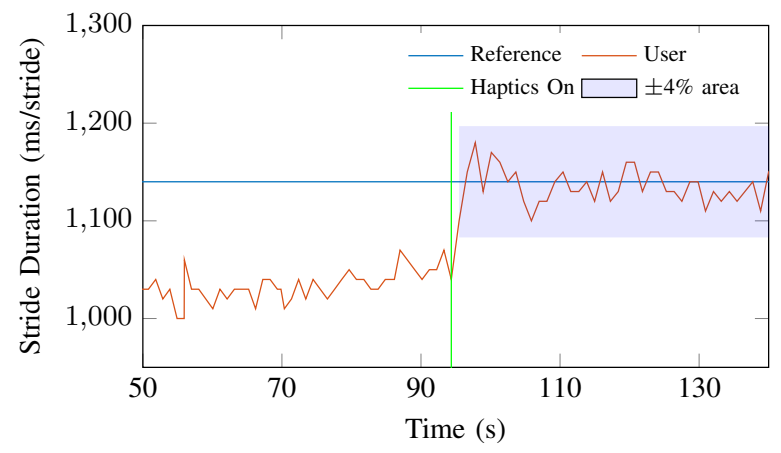

(b)

Fig. 6: Representative epochs for faster and slower cadence suggestion. In (a) the provided haptic rhythm has shorter period than the participant's baseline walking pace, and the user has to walk faster to match the external frequency. The green line represents the instant in which the haptic stimulation is activated, while the blue band highlights the tolerance band used to assess the alignment. Figure (b) is the symmetrical condition for slower cadence (in fact the stride duration reference increases wrt the baseline).

The secondary task was selected according to the requirement of low mental efforts, described in [26]. Subjects were asked to answer simple mathematical questions (double-digit sums and differences) on a smart-phone app and to walk at the same time, giving the same priority to the two tasks. We adopted the same procedure of the previous experiment: the first trial was meant to estimate the participants' baseline cadence, whereas the second trial was conducted with haptic stimulation enabled and the secondary task. Walking distance was $200 \mathrm{~m}$ in both cases and gait parameters investigated in the data analysis were the same as previous experiment.

Results: The analysis of comfortable cadence trials yielded baseline information about the gait parameters of our sample. In average, participants' stride duration was $1138 \pm 36 \mathrm{~ms}$, corresponding to a cadence of $52.72( \pm 2.19)$ strides/min. The inter-subject cadence variability, expressed in percentage, represents the $4.2 \%$ of the average value. This result is in line with the results of [35]. In that work, accelerometer signals recorded during the comfortable cadence walking of 60 subjects were analyzed. The authors reported a mean walking cadence of $53.54( \pm 3.87)$ strides/min, which corresponds to an average stride duration of $1120 \pm 64 \mathrm{~ms}$. The inter-subject cadence variability was $7.2 \%$. Anyway, those data were recorded while walking on regular and irregular surface, which would motivate the higher variability. We then calculated separately the stride duration variability (standard deviation) for each user, which mean value was $1.93 \%$, to assess the degree of physiological variability of human walking pace.

In order to discriminate changes in walking parameters due to haptic stimulation, we defined three tolerance bands ( $2 \%$, $4 \%$, and $6 \%$, corresponding to $\sigma, 2 \sigma$ and $3 \sigma$ ) around the reference stride duration subjects were asked to keep. In fact, we assume that cadence variations in the band reference 'stride duration $\pm 6 \%$ ' (three times the standard deviation) should be related to physiological variability, and higher misalignment may be due to the user being unable to follow the haptic rhythm. The tolerance bands were then used to investigate the amount of time, expressed in percentage of the trial duration since the beginning the synchronization with the haptic stimulation, in which subjects were able to follow the external pace given the acceptable error. This quantity is referred to as "alignment percentage".

In Table I we report the synchronization rate for each subject, calculated in the three tolerance bands. The median synchronization rates in the first experiment were $78.84 \%$, $99.28 \%$, and $100.00 \%$ for the $2 \%, 4 \%$ and $6 \%$ tolerances, respectively. The introduction of the secondary task lowered the overall synchronization rate: $47.50 \%, 85.92 \%$, and $99.80 \%$ were the median values extracted. We assessed through the Shapiro-Wilk's test that the data were not normally distributed, so we visually depicted data by means of box-plots in Fig. 5 and numerically using quartiles (reported in Table I).

To assess if the effect of vibrations was relevant, we compared data obtained during comfortable gait and haptic stimulation trials. A paired-samples t-test revealed a statistically significant mean difference in the stride durations recorded in the two conditions $(p=0.015)$. No outlier was detected. For both conditions, the assumption of normality was not violated, as assessed by Shapiro-Wilk's test $(p=0.195)$.

The same procedure was applied for the analysis of gait data recorded during the second experiment. Shapiro-Wilk's test confirmed the normal distribution of mean differences in stride duration per each subjects $(p=0.583)$, and the paired-sample t-test assessed that participants modified their stride duration also in presence of additional cognitive load $(p=0.04)$.

Discussion: Experimental results confirmed that humans can synchronize their step sequence to an external, constant rhythm provided through vibrotactile cues, with an error comparable to the natural cadence variability. Thus we can assume that it is possible to influence the participants' average cadence by asking them to voluntarily align to the provided rhythm.

The increase of cognitive load due to the secondary task did not have a relevant effect on the synchronization, which was achieved for most of the time by all the users, although the variability increased. Only one user could not successfully 


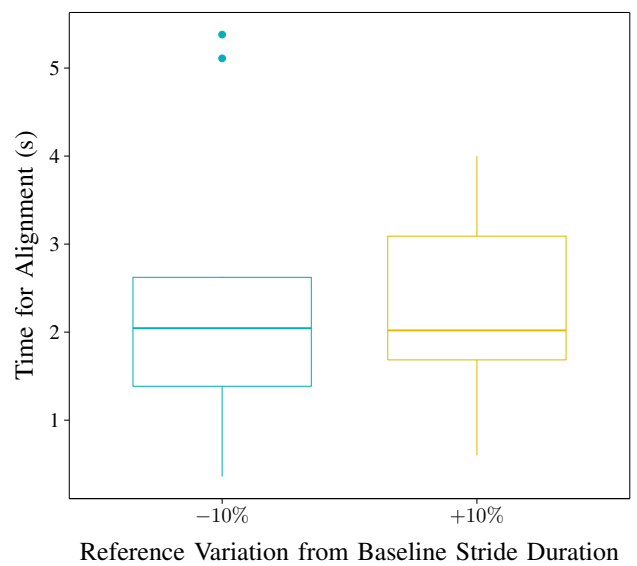

(a)

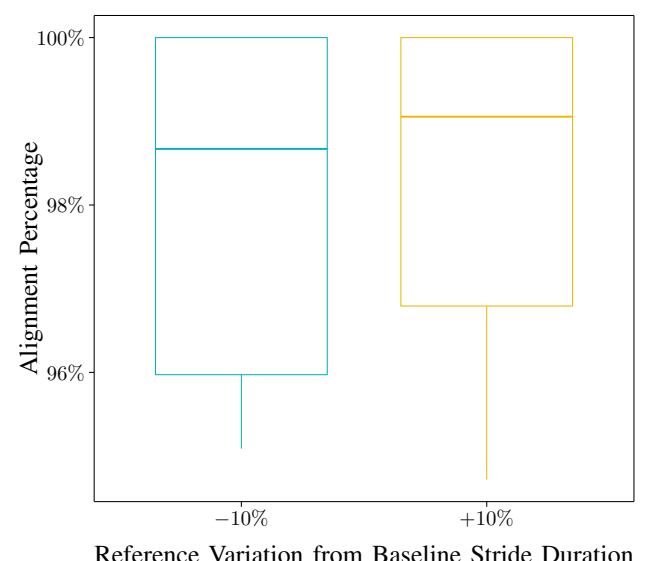

(b)

Fig. 7: The left boxplot (a) represents the distribution of times required by participants to align to the suggested pace, for $10 \%$ faster and slower cadence wrt to the baseline, respectively. The right boxplot (b) reports the distribution of trial time percentage (after the synchronization) during which participants' stride duration differed less than $4 \%$ from the suggested pace. In both cases, performance data show no significant difference due to increasing or decreasing stride duration.

\begin{tabular}{cccccc}
\hline \hline User & $\begin{array}{c}\text { Baseline } \\
\text { Stride Dur. } \\
(\mathbf{m s} / \text { stride })\end{array}$ & $\begin{array}{c}\text { Time for } \\
\text { Alignment } \\
(-10 \%)(\mathbf{s})\end{array}$ & $\begin{array}{c}\text { Alignment } \\
\text { Percentage } \\
(-10 \%)\end{array}$ & $\begin{array}{c}\text { Time for } \\
\text { Alignment } \\
(+10 \%)(\mathbf{s})\end{array}$ & $\begin{array}{c}\text { Alignment } \\
\text { Percentage } \\
(+10 \%)\end{array}$ \\
\hline U1 & 995 & 0.4 & 100.00 & 3.5 & 98.11 \\
U2 & 1146 & 1.3 & 100.00 & 1.5 & 100.00 \\
U3 & 1060 & 1.5 & 95.23 & 4.0 & 94.72 \\
U4 & 1021 & 2.6 & 95.09 & 3.1 & 100.00 \\
U5 & 1078 & 2.4 & 100.00 & 3.1 & 100.00 \\
U6 & 975 & 1.4 & 100.00 & 2.1 & 97.32 \\
U7 & 1107 & 2.6 & 97.34 & 1.8 & 100.00 \\
U8 & 1098 & 5.4 & 100.00 & 0.6 & 95.21 \\
U9 & 1100 & 1.7 & 97.66 & 1.9 & 100.00 \\
U10 & 1208 & 5.1 & 96.38 & 1.6 & 98.01 \\
\hline \hline
\end{tabular}

TABLE II: Data extracted from experimental data are listed in the table. The baseline stride duration represents the average cadence measured during the haptic-off trials. Time for alignment and Alignment percentages are then reported for fast $(-10 \%)$ and slow cadence condition $(+10 \%)$.

adapt to the suggested rhythm.

This experiment paved the way and defined some evaluation criteria for the other trials. To the best of our knowledge, literature lacks a clear and unanimous way of evaluating the human cadence synchronization with an external stimulus, therefore a straightforward choice was to use results of this experiment as a metric. The users' average stride duration variability during comfortable walking, in a regular surface without disturbance, is about $2 \%$. Thus, fluctuation around the mean value in the interval of $\pm 4 \%(2 \sigma)$ could be considered an appropriate interval for including the majority of the strides walked in a correct tempo. In the following experiments, $4 \%$ was used as reference to discriminate strides aligned and non aligned with the reference stride duration.

\section{B. Artificial constant reference}

Since the preparatory experiment (Sect. IV-A) only investigated the faster cadence condition, we enrolled 10 new participants (age $28.3 \pm 4.3,7$ males) to collect data on symmetrical pace variation. We replicated the setup of the previous case: participants were provided with the hardware, then their baseline cadence was acquired in a $100 \mathrm{~m}$ self-paced walk. Each subject was asked to perform two trials, during which they had to voluntarily synchronize their strides to a reference rhythm, $10 \%$ slower and faster than the baseline (the order was randomized). Trials were divided in $100 \mathrm{~m}$ of self-pace walking and $200 \mathrm{~m}$ of haptic-assisted walking. Stride times measured by the master anklet were logged by the system, and then compared with the reference to extract time to reach synchronization and alignment percentage (cfr. Fig. 6). In this experimental campaign we did not take into consideration the disturbance due to the secondary task, since we were interested only in the effects of the slower and faster external cadence.

Results: Stride duration data were processed to evaluate the time necessary to achieve synchronization with the haptic stimuli frequency, and to determine the deviation from the reference after the initial synchronization. The latter parameter was represented as the percentage of trial time during which the user's cadence drifted from the suggested cadence less than $4 \%$.

Shapiro-Wilk's test assessed that the distribution of times required by subjects to synchronize with the external cadence was normally distributed both for the fast $(p=0.09)$ and slow cadence $(p=0.78)$ conditions, while alignment percentages were not normally distributed in both cases ( $p=0.01$ for fast cadence, $p=0.01$ for slow cadence).

Mean time required to match the external stride duration were $2.44 \pm 1.63 \mathrm{~s}$ for fast condition and $2.31 \pm 1.05 \mathrm{~s}$ for slow condition, while median alignment percentages were $98.8 \%$ 


\begin{tabular}{cccc}
\hline \hline User & $\begin{array}{c}\text { Baseline } \\
\text { Stride Duration } \\
\text { (ms/stride) }\end{array}$ & $\begin{array}{c}\text { Time for } \\
\text { Alignment }\end{array}$ & $\begin{array}{c}\text { Alignment } \\
\text { Percentage }\end{array}$ \\
\hline U1 & 1206 & 4.0 & $\%$ \\
U2 & 1336 & 4.7 & $100 \%$ \\
U3 & 955 & 3.6 & $99 \%$ \\
U4 & 998 & 1.0 & $97 \%$ \\
U5 & 905 & 3.8 & $99 \%$ \\
U6 & 920 & 0.3 & $98 \%$ \\
U7 & 879 & 4.8 & $99 \%$ \\
U8 & 1006 & 2.9 & $98 \%$ \\
U9 & 1060 & 4.2 & $95 \%$ \\
U10 & 986 & 5.6 & $100 \%$ \\
U11 & 881 & 4.5 & $99 \%$ \\
U12 & 885 & 4.5 & $99 \%$ \\
U13 & 980 & 1.5 & $99 \%$ \\
U14 & 1009 & 4.3 & $98 \%$ \\
U15 & 1168 & 1.5 & $94 \%$ \\
U16 & 1077 & 5.5 & $99 \%$ \\
U17 & 961 & 2.1 & $98 \%$ \\
U18 & 1058 & 2.5 & $100 \%$ \\
U19 & 1297 & 2.7 & $99 \%$ \\
U20 & 1251 & 5.4 & $99 \%$ \\
\hline \hline
\end{tabular}

TABLE III: Artificial Leader. For each user we report data from the experimental validation. The first column details the user's comfortable cadence (i.e., cadence without haptic suggestion). In the second column we report the total time needed to align the actual cadence to the displayed one. The last column describes the percentage of time in which the subject was aligned with the suggested rhythm.

and $99.0 \%$ respectively. Boxplots in Figs. $7 \mathrm{a}$ and $7 \mathrm{~b}$ visually describe data, that are also listed in Table III.

Paired t-test conducted on time to alignment values revealed no statistical difference between the two distributions $(t(9)=$ $0.14, p=0.89)$. No statistical test was conducted on alignment percentages because visual representation showed very small difference between the two distributions.

Discussions: This experiment was aimed to assess performance asymmetries during faster and slower pace conditions. Experimental results suggest that participants managed to tune their walking pace to the external rhythm for a large portion of the trial duration, regardless of the sign of the cadence variation. The statistical analysis of time to reach synchronization also did not evidence significant differences between conditions. For these reasons, we expect that participants abilities in synchronizing is not asymmetrically biased by the sign of the cadence variation. Forthcoming experimental procedures investigate in detail human acceptance of fast varying gait rhythms, thus broadening the study on the participants' proficiency in aligning to faster and slower paces.

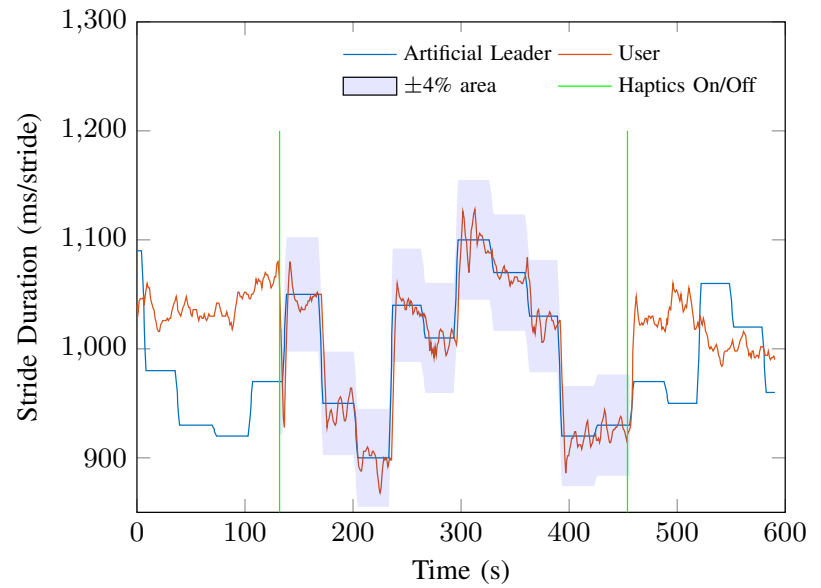

Fig. 8: Artificial Leader representative trial. The participant was tasked to align his cadence with the one proposed via the wearable haptic devices. The user started the trial walking at his most comfortable gait. After 200 meters (about 130 seconds) the haptics were automatically turned on and the user was able to feel the vibrotactile stimulation. The stimulation continued for 400 meters, than the interfaces were turned off. The participant walked for additional 200 meters at his most comfortable cadence. Green lines identify the time-points in which haptics were turned on and off. The user's cadence is depicted with a red line, whereas the blue line and the surrounding violet area represent the suggested rhythm and the $\pm 4 \%$ interval, respectively.

\section{Artificial leader}

In this experiment we examined the human capability in entraining to a time-varying rhythm generated by an algorithm. This is a common modus operandi in training and rehabilitation bouts, sportspeople and patients have to follow an external pace with time-varying frequency in order to improve (or recover) physical abilities. We named this methodology leader-follower, borrowing the idea from robotics, because the follower is asked to align his step sequence to the haptic rhythm displaying the leader's walking cadence (in this case simulated). Twenty participants (age $29.8 \pm 5.3,14$ males) have been recruited for this phase.

The experimental setup was composed by two anklets, one of which equipped with the force sensor for recording the stride sequence, headphones reproducing white noise, and a smart-phone with the $a d-h o c$ application. Each subject performed a single trial composed of three phases. In the first phase the participant was instructed to walk at his/hers comfortable pace for $200 \mathrm{~m}$, to record baseline cadence. In the second phase the user was asked to align to the pace provided through haptic stimuli, for $400 \mathrm{~m}$. In the last phase, vibrations were turned off and the subject continued walking for $200 \mathrm{~m}$ at his comfortable pace. A dedicated piece of software simulated the leader's cadence updates that, though the server, instructed the app to vary the vibration frequency. A new reference stride duration was randomly selected every 30 seconds in the interval $900-1100 \mathrm{~ms}$ (average stride duration is $1 \mathrm{~s}$ ).

We selected a $30 \mathrm{~s}$ update time to analyze the stability of 


\begin{tabular}{cccccccc}
\hline \hline Leader & Follower & $\begin{array}{c}\text { Leader B } \\
\text { Stride Duration } \\
(\mathbf{m s} / \text { stride })\end{array}$ & $\begin{array}{c}\text { Follower B } \\
\text { Stride Duration } \\
(\mathbf{m s} / \text { stride })\end{array}$ & $\begin{array}{c}\text { Mean H } \\
\text { Stride Duration } \\
(\mathbf{m s} / \text { stride })\end{array}$ & $\begin{array}{c}\text { STD H } \\
\text { Stride Duration } \\
(\mathbf{m s} / \mathbf{s t r i d e})\end{array}$ & $\begin{array}{c}\text { Time for } \\
\text { Alignment }\end{array}$ & $\begin{array}{c}\text { Alignment } \\
\text { Percentage }\end{array}$ \\
\hline U1 & U2 & 1023 & 1141 & 1022 & 19 & 2.0 & $97 \%$ \\
U3 & U4 & 1145 & 1035 & 1151 & 21 & 4.4 & $99 \%$ \\
U5 & U6 & 1025 & 1136 & 1037 & 17 & 3.5 & $94 \%$ \\
U7 & U8 & 1014 & 1081 & 1017 & 16 & 3.2 & $98 \%$ \\
U9 & U10 & 1050 & 1082 & 1042 & 18 & 3.9 & $96 \%$ \\
U11 & U12 & 1113 & 1106 & 1110 & 17 & 3.2 & $99 \%$ \\
U13 & U14 & 1020 & 1070 & 1024 & 16 & 2.5 & $95 \%$ \\
U15 & U16 & 1086 & 1030 & 1078 & 20 & 3.9 & $98 \%$ \\
U17 & U18 & 1055 & 1101 & 1059 & 23 & 3.0 & $94 \%$ \\
U19 & U20 & 1018 & 1081 & 1030 & 20 & 3.5 & $98 \%$ \\
\hline \multicolumn{2}{c}{ AVERAGE } & & & & $\mathbf{1 8 . 7}$ & $\mathbf{3 . 3 1}$ & $\mathbf{9 6 . 8 \%}$ \\
\hline \hline
\end{tabular}

TABLE IV: Human Leader. The table details data regarding the experiment with the human leader, where $\mathbf{B}$ and $\mathbf{H}$ stand for baseline and haptic-on condition, respectively. Mean cadences estimated in the first part of the trial (without haptics) are reported for both the users, and compared to the average walking rhythm during the phase with the haptic stimulation (Mean $\mathrm{H})$. It is important to notice that the standard deviation of the mean cadence during the phase with haptic stimulation is comparable with the variability registered in the comfortable cadence walking.

gait cadence after each variation and the number of strides necessary to adapt to the new stride frequency. The second phase of the experiment in average lasted 4 minutes, resulting in at least 7 cadence variations. A representative trial is reported in Fig. 8

From each trial we examined: i) follower's comfortable cadence before vibrational cueing, ii) strides needed by the follower to align his gait with the proposed cadence (considering a $4 \%$ tolerance), iii) percentage of time the follower is aligned with the suggested gait.

Results: The primary aim of this experiment was assessing whether humans could align their cadence to a time-varying frequency. We calculated for each subject the percentage of time in which stride duration was in the range reference stride duration $\pm 4 \%$ during the phase with haptic cues. All followers were able to align to the leaders' rhythm for more than $94 \%$ of the trial time. Data of the trials are reported in Table III.

The average number of strides necessary to adapt to the new cadence is $2.2 \pm 1.2$. In particular, an asymmetry was observed between increasing and decreasing stride duration: the number of strides necessary to achieve a misalignment lesser than $4 \%$ was $1.1 \pm 0.7$ and $3.1 \pm 1.9$ strides for slower and higher frequency variations, respectively. For variations of the reference stride duration below $4 \%$ in most cases there was no transient in aligning to the new cadence.

Discussion: Outcomes of the test revealed that participants could easily adapt to cadence variations, especially if the difference was small. In fact, considering the human temporal resolution and physiologic variability of gait, the user may not even notice small variations (in the order of $20 \mathrm{~ms}$ ). These results allow to study the synchronization of human cadence with external rhythms which vary fast, but with limited oscillations, as in the case of human gait. For variations greater than $4 \%$, results show that users react quickly to cadence increase (i.e., smaller stride duration), probably by making smaller steps to restore the synchronization with the external rhythm, whereas it seems more difficult to rapidly reduce the pace (i.e., increase the stride duration). The last phase of recording without haptics is not studied quantitatively; we plot it to demonstrate the effect of the haptic stimulation. In fact, after the vibrations were turned off, the self-selected stride duration was restored to the baseline value.

\section{Human leader}

The results obtained in the previous experiment encouraged the assumption that humans can adapt with ease their walking cadence to time-varying rhythms if the variability is limited (assuming the human cadence physiological variability as boundaries). In this experimental session the follower is provided with haptic stimuli replicating the human leader's cadence. We stress that in this experiment the leader could not feel the follower by any means.

Twenty subjects (age 27.9 $\pm 6.1,12$ males) took part in this phase. The experimental setup for each participant was composed by two haptic interfaces, one of which equipped with the force sensor for recording the stride sequence, headphones reproducing white noise, a smart-phone with the ad-hoc app. The 20 participants, randomly labeled from U1 to U20 for convenience, were arranged in couples. Each couple performed one trial, the role of leader and follower was selected randomly at the beginning of the trial. In the first phase of the trial, both participants were asked to walk at their comfortable cadence for $200 \mathrm{~m}$, to record gate parameters in the baseline condition. In the second phase the follower received haptic stimuli replicating the leader's gate cadence, to which he has been instructed to adapt. The leader was not notified about the beginning of the second phase, and continued walking at his own pace. The anklets were used by the leader exclusively to record the strides duration, while vibro-motors never activated. 


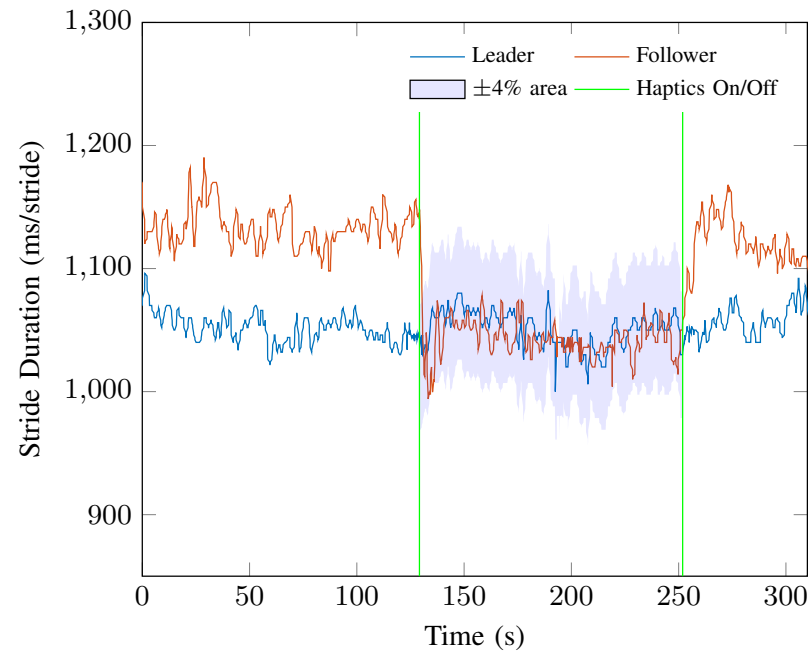

Fig. 9: Human Leader representative trial. The follower was tasked to align the walking cadence with the leader's one, transmitted via the wearable haptic devices. The users started the trial walking at their comfortable pace. After 200 meters (about 120 seconds) the follower's haptics were automatically turned on to enable the vibrotactile stimulation, while the leader continued walking at self-selected pace. After 200 meters, the follower's anklets were turned off and the participants walked for additional 200 meters without haptic suggestions. Green lines identify the time follower-side haptics were turned on and off. The user cadence is depicted with a red line, whereas the blue line and the surrounding violet area represent the suggested rhythm and the $\pm 4 \%$ area, respectively.

After $200 \mathrm{~m}$, haptics were turned off and the last phase began, during which subjects were instructed to walk for $200 \mathrm{~m}$ at their comfortable cadence. A representative trial is reported in Fig. 9 .

From each trial we estimated: $i$ ) comfortable cadences before haptic cueing, ii) time needed by the follower to align to the leader's cadence (calculated from the initial activation of the haptic devices to the reaching of the desired cadence, considering the $4 \%$ error bound), iii) percentage of time follower is following the leader tempo (defined as the follower's cadence $\pm 4 \%$ ).

Results: Experimental results (detailed in Table IV) show that all the followers succeeded in aligning their walking cadence to the leader's for more than $90 \%$ of the time, assuming an acceptable oscillation of $4 \%$ around the reference pace. The average time required to align with the leader was $3.31 \pm 0.69$ seconds.

Discussion: In this experimental session we evaluated the human capability in adapting the walking cadence to a fastvarying pace displayed through vibrations. Experimental results show that cadence oscillations due to natural variability do not impede the entraining with haptic cues.

These results open a wide range of applications in which a leader guides one or more followers, as in training and rehabilitation. A more comprehensive discussion on possible future research directions is reported in VII-B Moreover, these results provide the last prerequisites for hypothesizing and testing the mutual cadence alignment, i.e. remote social walking, referred in the following as 'peer-to-peer'.

\section{E. Peer-to-peer}

This experimental session represents the last piece of the remote social walking step-wise validation. Once the capability in following an external rhythm was assessed, we tested bilateral transmission of cadence through vibrotactile interfaces to connect two people walking far from each other. Our aim is testing if the system we developed can be successfully used to achieve the cadence synchronization between two users without direct interaction. An assumption we had to make was asking participants to voluntarily align to the partner's cadence, but still keeping a step frequency close to their comfortable one. In fact, the group walking (or social walking) condition is replicated if the participants agree on a common pace comfortable for everyone. As a consequence, in this experiment the users did not receive strict guidelines, they had to 'negotiate' with the partner. Although the psychological aspect plays a relevant role in the achievement of the consensus, it will be studied in a future work. In fact, before studying how people agree on a common rhythm, we need to validate the proposed system and assess if and how the cadence alignment takes place. Thus, in this work we study temporal gait parameters to investigate the system features and capabilities.

Twenty participants were enrolled for the experimental session (age 28.1 $\pm 5.4,8$ males), randomly labeled from U1 to U20, and arranged in couples. All the users took part in a previous experiment, at least. The experimental setup for each subject was composed by two haptic interfaces, one of which equipped with the force sensor for recording the stride sequence, headphones reproducing white noise, a smart-phone with the ad-hoc app.

Trials were composed by three phases: users were asked to start from predefined positions and walk at their comfortable pace along different paths for $200 \mathrm{~m}$. In the second phase haptic stimuli representing the partner's cadence were delivered to each participant, who was instructed to adapt to the received rhythm and, simultaneously, try to pull the partner toward his own pace. After both participants walked $200 \mathrm{~m}$, the haptic stimulation was turned off and the users walked at their own cadence for 200 meters. A representative trial is reported in Fig. 10

In order to give a quantitative evaluation of the effectiveness of our system, here we defined the concept of cadence alignment: a user's stride is aligned with the partner's if the duration of the current stride is in the interval partner's last stride duration $\pm 4 \%$. In this last experiment we analyzed: $i$ ) comfortable cadences, $i i$ ) variation of the average cadence for each user during the haptic stimulation phase (with respect to the comfortable cadence), iii) time needed for reaching the alignment, $i v$ ) percentage of time users strides were aligned.

Results: All users, with exception of two, varied their average walking cadence during the phase with haptic stimulation, as visible in Table $\mathrm{V}$ The average stride duration variation with respect to the comfortable pace was $4.0 \%$. In all but two 


\begin{tabular}{cccccccccc}
\hline \hline User1 & User2 & $\begin{array}{c}\text { User1 B } \\
\text { Stride Duration } \\
(\mathbf{m s} / \text { stride })\end{array}$ & $\begin{array}{c}\text { User2 B } \\
\text { Stride Duration } \\
(\mathbf{m s} / \text { stride })\end{array}$ & $\begin{array}{c}\text { Mean H } \\
\text { Stride Duration } \\
(\mathbf{m s} / \mathbf{s t r i d e})\end{array}$ & $\begin{array}{c}\text { STD H } \\
\text { Stride Duration } \\
(\mathbf{m s} / \mathbf{s t r i d e})\end{array}$ & $\begin{array}{c}\text { Time for } \\
\text { Alignment } \\
(\mathbf{s})\end{array}$ & $\begin{array}{c}\text { Alignment } \\
\text { percentage }\end{array}$ & $\begin{array}{c}\text { User1 } \\
\text { Variation }\end{array}$ & $\begin{array}{c}\text { User2 } \\
\text { Variation }\end{array}$ \\
\hline U1 & U2 & 1082 & 1158 & 1136 & 18 & 3.6 & $95 \%$ & $5 \%$ & $2 \%$ \\
U3 & U4 & 1060 & 1002 & 1023 & 25 & 5.2 & $96 \%$ & $4 \%$ & $2 \%$ \\
U5 & U6 & 1139 & 1136 & 1190 & 32 & 4.2 & $96 \%$ & $4 \%$ & $5 \%$ \\
U7 & U8 & 1004 & 1070 & 1083 & 25 & 2.8 & $91 \%$ & $7 \%$ & $1 \%$ \\
U9 & U10 & 1039 & 982 & 1046 & 31 & 1.1 & $92 \%$ & $1 \%$ & $6 \%$ \\
U11 & U12 & 1067 & 1047 & 1090 & 35 & 5.6 & $93 \%$ & $2 \%$ & $4 \%$ \\
U13 & U14 & 996 & 1167 & 1087 & 33 & 11.6 & $99 \%$ & $8 \%$ & $7 \%$ \\
U15 & U16 & 1102 & 1119 & 1145 & 33 & 7.3 & $86 \%$ & $4 \%$ & $2 \%$ \\
U17 & U18 & 1032 & 1103 & 1067 & 22 & 4.9 & $99 \%$ & $3 \%$ & $3 \%$ \\
U19 & U20 & 1082 & 1197 & 1116 & 24 & 2.9 & $98 \%$ & $3 \%$ & $7 \%$ \\
\hline AVERAGE & & & $\mathbf{2 8}$ & $\mathbf{4 . 9}$ & $\mathbf{9 4 . 5 \%}$ & $\mathbf{4 . 1 \%}$ & $\mathbf{3 . 9 \%}$ \\
\hline \hline
\end{tabular}

TABLE V: Peer-to-peer. The table details data regarding the final experiment, where $\mathbf{B}$ and $\mathbf{H}$ stand for baseline and haptic-on condition, respectively. Mean cadences estimated in the first part of the trial (without haptics) are reported for both the users, and compared to the average walking rhythm during the phase with the haptic stimulation (Mean $\mathrm{H}$ ). Also in this experiment is important to notice that the standard deviation of the mean cadence during the phase with haptic stimulation is comparable with the variability registered in the comfortable cadence walking.

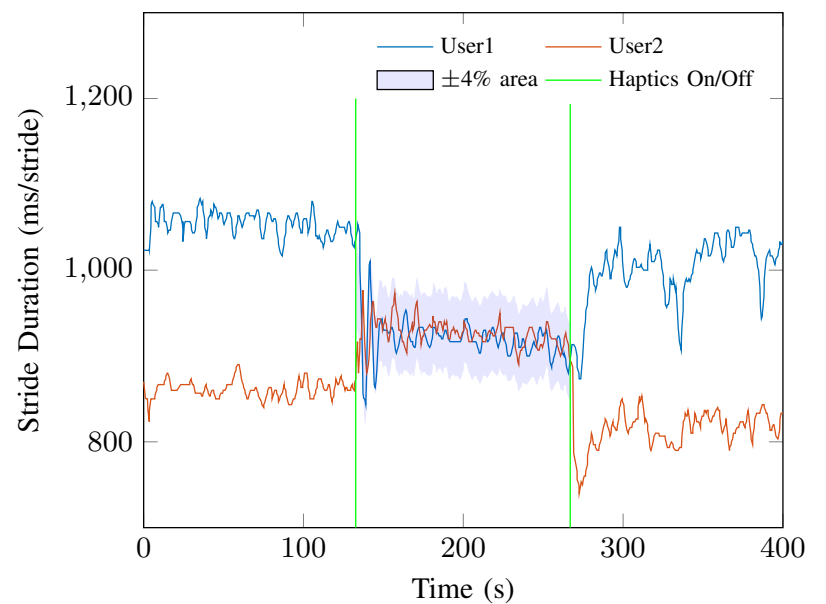

Fig. 10: Peer-to-peer representative trial. The participants were tasked to tune their own gait cadence with the partner's rhythm, displayed by the anklets. The users started the trial waking at their comfortable cadence. After 200 meters (about 120 seconds) the haptics were activated and both users were able to feel the partner's cadence for 200 meters. Then the interfaces were turned off again and the participants walked for additional 200 meters at their comfortable cadence. Green lines identify the time-points in which haptics were turned on and off. The users' cadences are depicted respectively with red and blue lines, the surrounding violet area represents the $\pm 4 \%$ area of the mean computed at each timestamp.

cases the participants agreed on a common cadence which was intermediate between the two comfortable cadences. The average time to reach the cadence alignment was $4.92 \pm 2.91$ seconds. After the beginning of the alignment, on average, the participants maintained a similar gait frequency (in the limits of $4 \%$ ) for the $94.5 \pm 4.1 \%$ of the time.
Discussion: As shown in Table V, the effect of haptic stimulation is evident and results demonstrate the effectiveness of the system. All the participants changed their walking pace according to the partners' stride duration after the stimulation was activated, and immediately moved back to their comfortable cadence after the haptic phase. It is worth pointing out that the time for aligning the cadence to the partner's is higher than the ones observed in the Leader-Follower case. This is probably a consequence of the fact the users try to follow the partners' cadence, resulting in a transient during which the users' stride durations oscillate. In addition, for the majority of the trial, we observed a greater oscillation at the beginning of the haptic cueing, followed by a constant reduction. This is characteristic for a system with an inertia following a reference. Although the study of psychological aspects is not in the focus of this work, we can make two considerations:

i) in most of the trials the participants aligned their gait cadence on a common rhythm which was close to the mean value of the comfortable cadence of the two users;

ii) in two trials participants achieved the consensus, but they aligned on a cadence which was close to the comfortable cadence of one of the participants. A possible explanation is that one user may not have fully understood the experimental protocol.

\section{HUMAN BEHAVIORS AND PERFORMANCE CORRELATION ANALYSIS}

The presented experimental validation and the following results discussion can be enriched by analyzing the correlation between the participants' self-pace at baseline and their ability to align the walking cadence to the reference pace. In particular, we searched for possible relationships between subjects comfortable cadence, suggested rhythm, and success in synchronizing. We started by evaluating scenarios 
with artificial rhythms, both in case of constant (Sect. IV-B) and variable reference cadence (Sect. IV-C). In the former scenario, we tested the presence of a relationship between the suggested pace and alignment performance. The difference between stride duration suggested and participants' baseline cadence was correlated with the time required to synchronize with the external stimuli, and with the percentage of task time during which the participants' stride duration was comparable with the haptic stimulation period. Since synchronization percentages values were not normally distributed, we resorted to Spearman's correlation tests, while Pearson's test was used for time to alignment. The tests revealed no significant relationship of baseline both with time $(p=0.22)$ and alignment percentage $(p=0.86)$. For what concerns the 'Artificial Leader' data, the considered values were not normally distributed so Spearman's rank-order correlation tests were run to assess the relationship between baseline and performance. Results of the tests show that there was no statistically significant correlation between comfortable stride duration and percentage $(p=0.117)$. Similarly, there was no statistically significant correlation between baseline and time for alignment $(p=0.794)$.

This result is not surprising, because the displayed stride duration was not constant and was updated every $30 \mathrm{~s}$ in the range going from 900 to $1100 \mathrm{~ms} / \mathrm{stride}$. In fact, this experiment was aimed to assess the behaviour of participants when facing cadences varying in a wide range. The fact that all participants managed to align to the external rhythm with no dependence on the baseline gait parameters may prove that, as long as the suggested cadence is selected inside a feasible range, the human can successfully adapt his own walking pace. The time to achieve the alignment instead was calculated as the sum of the synchronization time after each cadence variation, thus it depends also from the randomness factor.

Then we took into consideration the social aspect and the users' response in following a partner. Outcomes from the experiments described in Sect. III-D and Sect. III-E were analyzed. For what concern the 'Human leader' scenario, we evaluated the relationship between the time to reach the synchronization, the percentage of the trial in which the leader was aligned with the master, and the difference between leader's and follower's stride duration. All the considered variables were normally distributed, as assessed by Shapiro-Wilk's test (difference $p=0.391$, alignment percentage $p=0.164$, and time to alignment $p=0.965$ ). The Pearson's product-moment correlation revealed no significant correlation between walking pace difference and alignment percentage $(p=0.128)$. On the contrary, Pearson's product-moment correlation between initial stride duration difference and time to alignment was statistically significant $(r=0.714, p=0.02)$.

As in the 'Artificial Leader' experiment, the lack of relationship between baseline cadence and alignment performance may imply that the self-selected pace does not affect the synchronization percentage. On the other hand, the significant correlation of baseline pace with the alignment time may be due to the fact that accommodating to a farther rhythm takes longer. This aspect is interesting on the perspective of defining effective strategies to facilitate the alignment between two or more participants: instead of providing the raw partner's cadence, it may be smoothed to avoid oscillations during transient.

Similar results were collected for data in the 'peer-to-peer' experiment (Sect. III-E). The same metrics were exploited to evaluate the correlation between users' pace and performance. Shapiro-wilk's test assessed normality distribution for stride duration difference $(p=0.255)$, alignment percentage $(p=0.546)$, and time to achieve alignment $(p=0.249)$. Pearson's product-moment correlation revealed no statistically significant relationship between the difference in initial gait cadence and alignment percentage $(p=0.081)$, neither between cadence deviance and time to reach the common stride duration $(p=0.263)$. While the former result is in line with the one obtained from 'Human Leader' data, the latter is in contrast. Further experiments are required to address this matter, but we hypothesize that the two participants' efforts in aligning their cadence may generate non-linear dynamics.

\section{QUalitative RESUlts AND USERS' FEEDBACK}

Similarly to [36], at the end of the 'peer-to-peer' experiment participants were asked to fill a questionnaire comprising four multiple-choice and one open question about personal impressions and suggestions. The aim of the questionnaire was investigating the effectiveness of the system with a qualitative approach.

It is worth pointing out that all the subjects involved in the survey participated in at least two experiments. The first question was about the spontaneity in aligning to the external rhythm provided by the haptics. With the second question we evaluated the ease of use of the vibro-tactile anklets. The following topic under investigation was the social side of the proposed work: we asked subjects opinion on the system transparency, i.e., whether the stimulation resembles a human walking cadence. Finally, we evaluated the impressions of walking with a remote companion.

The list of questions is reported in the following.

Q1: Did aligning to the vibrations come naturally to
Q2: Could you align with ease to the rhythm received?
Q3: Do you think that the vibrations you received could
Q4: Did you perceive your partner's telepresence?

Answers were entered on a Likert scale with range 1-7, where 1 represented 'Strongly Disagree', and 7 'Fully Agree'.

Results: Answers to the questionnaires are reported in what follows in terms of mean \pm standard deviation. The subjects' average ratings were $4.3 \pm 1.5,4.8 \pm 1.3,5.7 \pm 1.1$, and $5.9 \pm 1.1$, respectively for questions Q1, Q2, Q3, and Q4. A graphical representation of the users' answers is reported in Fig. 11 .

Discussion: The analysis of the multiple choice questions confirms that, for almost all participants, our system is an effective mean to transmit walking cadence. Vibrational cues are generally perceived as an easy and intuitive way to 'feel' 


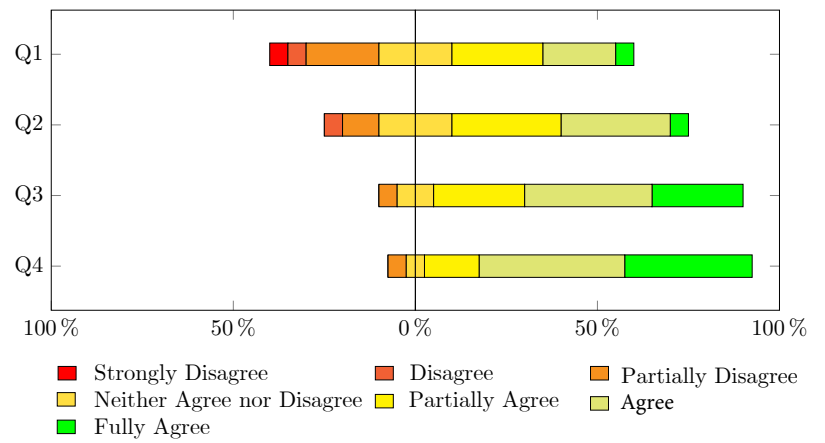

Fig. 11: Likert scale data for the proposed questionnaire. For each question the percentage of answers is reported.

the presence of the remote companion. Although most of the users felt the system mechanism to convey the gait cadence as natural, some did not agree on its intuitiveness. The cadence alignment takes place and the great majority of the users are motivated to adapt to it, even though roughly one out of four found it hard to achieve synchronization.

Moreover, the answers to the open questions revealed that not only the emotive aspect incentives the alignment, but also that synchronizing to the vibrations is satisfying.

As a conclusive assessment we evaluated the possible correlation between the users' performance and the correspondent questionnaire responses. A global score was calculated for each participant as the sum of the four questionnaire ratings. For what concerns the variation of the walking rhythm during the 'peer-to-peer' experiment (see Sect. IV-E and Table V), we did not select the users' cadence variation from the baseline value, because it does not consider the partners' behaviour during the experiments. Instead, we used the difference between user's and partner's cadence variation during the task. For instance, the couple U7-U8 (Table V) has an average variation of $4 \%$, but $\mathrm{U} 7$ modified his cadence by $7 \%$, while the U8's change was only $1 \%$. On the contrary, U5 and U6 average cadence modification was $4.5 \%$, with a slight difference among them $(1 \%)$. In those cases, cadence variations were $+6 \%$ and $-6 \%$ respectively for $\mathrm{U} 7$ and $\mathrm{U} 8$ (obtained as variation $_{U 7}-$ variation $_{U 8}$ and variation $_{U 8}-$ variation $\left._{U 7}\right)$, and $-1 \%$ and $+1 \%$ for U5 and U6.

Firstly, we assessed through the Shapiro-Wilk's test the normality of data. While the users' ratings and the percentage variations of the user' gait were normally distributed ( $p=$ 0.358 and $p=0.977$ ), alignment percentage failed ShapiroWilk's test $(p=0.037)$. Pearson's product-moment correlation was run to assess the relationship between questionnaire rates and user's cadence variation. There was a statistically significant positive correlation between percentage variation in modifying the walking cadence and answers in the survey $(r=0.701, p=0.01)$. The Spearman's test between trial aligment percentage and questionnaire ratings revealed no statistically significant correlation $(p=0.204)$.

Users' rating are not linked with the task performance (i.e., walking and reaching a common rhythm), as already suggested by correlational tests in the previous section. On the other hand, the correlation coefficient expresses a strong relationship $\left(r^{2}=0.49\right)$ between questionnaire ratings and participants' relative cadence variations after the synchronization with partners. Although correlation does not imply causality, we hypothesize that participants who did accomodate to the partners' rhythm succesfully had rated their experience as positive, while users who felt uncomfortable with the haptic stimulation mainly expressed low scores. This assumption lays the basis for the next projects, were participants' behaviour will be investigated as a factor to achieve cadence alignment with multiple partners. Moreover, we need to test whether training affects the users' acceptance of our system.

\section{CONCLUSIONS}

\section{A. Summary}

In this paper a system for social remote walking was presented and incrementally tested in each of the aspects comprising its global functioning. After designing the technological parts (hardware and software), and performing engineering testing, a first experimental session confirmed that humans can follow a time varying artificial rhythm perceived via anklet vibrations. We then assessed that the tracking performances are retained when the virtual reference is replaced with a human gait cadence with a dedicated set of experiments. Finally, we obtained experimental evidence that two humans, walking simultaneously but not in each other proximity, can synchronize their gait cadence when perceiving the companion's walking rhythm using our system.

\section{B. Future research directions overview}

The presented results pave the way for numerous interesting research directions that will be the subject for future works. We briefly list the most attracting.

The paper mainly focused on presenting the haptic system and testing its effectiveness in allowing mutual gait cadence influence in humans. Following that confirmation, we are ready to extend results to more various population including older adults.

Even if this work focused on its social aspect, the presented system may also be used by a single walker to have a personal stimulus and track a cadence profile. Such profile may come by a previous personal run, or by a friend's one; additionally it can also be prepared by a personal trainer. In a similar fashion, rehabilitation scenarios can be designed so that patients can exercise under supervision.

In presence of relevant differences in height or training condition, the synchronization may be difficult to achieve. In this case, it would be wise to investigate whether a scale factor would help to agree a common, even if different, pace cadence while retaining the feeling of 'walking together'. Note that gait using a scale factor is not feasible while walking side by side.

Our study can be extended to a group of more than two humans. Game Theory provides numerous models that could potentially be suitable for the interpretation of the occurring group dynamics. Among the relevant indexes the synchronization and consensus of gait cadence are the most attracting. 
One may also investigate different strategies to display information though vibrations, or new algorithms to facilitate synchronization (for two or more users) tailored on scenarios.

Finally, we believe that our results on remote social walking can be extended to jogging and running.

\section{APPENDIX: INTERFACING AND USING NIKE+IPOD KIT}

In this section we briefly detail the interfacing procedure for using the Nike+iPod sensor for customized application. The kit (approx. 29\$ (USD)) contains two modules: a tiny sensor to be placed in the shoe and a receiver to be used with iPod. When the user walks or runs, the piezo-sensor estimates and wirelessly transmits information about the user's gait to the receiver. Following the result presented in [37], we modified an iPod female connector by soldering wires from the serial pins on the iPod connector to our adapter, adjusted the voltage accordingly, and powered with $3.3 \mathrm{~V}$. We then plugged a Nike+iPod receiver into our female connector replacing the Ipod with a PC running an $a d-h o c$ developed software. This caused the receiver to start sending packets over the serial connection to our computer, allowing us to monitor the measured cadence. Acosta et al. in [38] and Kane et al. in [39] validated the accuracy of the Nike+ Wireless Sport Kit to estimate pace $(\mathrm{min} / \mathrm{km})$, and distance $(\mathrm{km})$ during treadmill walking and running. Results showed that the Nike+ device overestimated the speed of level walking at $3.3 \mathrm{~km} / \mathrm{h}$ about $20 \%$, underestimated the speed of level walking at $6.6 \mathrm{~km} / \mathrm{h}$ by $12 \%$, but correctly estimated the speed of level walking at $4.9 \mathrm{~km} / \mathrm{h}$, and level running at all speeds $(p<0.05)$. Similar results were found for distance estimation. Starting from the preliminary results presented in [37] we developed a device for receiving and decoding messages from th Nike+ sensor. We designed and build an ad-hoc PCB for connecting the receiver with an Arduino based micro-controller. We can split the developed code in two main parts. The former acquires information from the sensor and sends the computed cadence to a remote server using internet, the latter receives the information about the partner rhythm and activates the motors correspondingly. Two serial communications were created in order to communicate at the same time with the sensor and the smartphone. The communication between the pedometer and our system starts sending a header packet of 8 byte. This packet puts the sensor in active mode and the stream of data is enabled. We observed that the sensor streams a packet of 34 bytes every seconds. We collected and analyzed several packets from multiple sensors, noticing some common bytes. A representative packet is the following: $\mathrm{FF} 55 \mathrm{EE} 09$

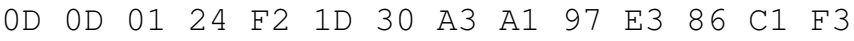

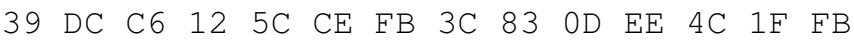
$\mathrm{F} 8$ 38. We discovered that $\mathrm{FF} 55$ is the packet header, and the payload starts with $1 E$ O 9 OD OD 01 for all the sensors and all packets. The packet continues with 27 bytes. The first 26 bytes carries all the information estimated by the pedometer, such us walking steps, running steps, sensor ID, lifetime walking and running miles, etc. The last byte is used as a check-sum to validate or discard received packets. We tested all the possible combination of packet bytes and checksum type and we found that the last byte is a $8 b i t 2 s$ Complement checksum. The 26 bytes payload are decoded using a library based on the work done by Grinberg [40]. All the sensors use the same radio frequency, and a packet per second is sent regardless the presence of a request or ack from the receivers, thus to use multiple Nike+ we process packet only if the descrambled serial number matches the one associated to the user. One per second Arduino receives the total amount of walked (or run) steps. We exploit this incremental measures to compute the cadence i.e., the number of steps per minute. A moving average with time window of 5 seconds is used to have good compromise between response time and smoothness. As soon as a change in the cadence occurs, the smart-phone (or smart-watch) is notified.

\section{REFERENCES}

[1] S. S. Wiltermuth and C. Heath, "Synchrony and cooperation," Psychological science, vol. 20, no. 1, pp. 1-5, 2009.

[2] J. Hannah, "African dance and the warrior tradition," Journal of Asian and African Studies, vol. 12, no. 1-4, pp. 111-133, 1977.

[3] B. Ehrenreich, Dancing in the streets: A history of collective joy. Macmillan, 2007.

[4] G. St John, "'connectedness' and the rave experience: rave as new religious movement?" in Rave culture and religion. Routledge, 2004, pp. 101-122.

[5] S. Hanson and A. Jones, "Is there evidence that walking groups have health benefits? a systematic review and meta-analysis," British Journal of Sports Medicine, vol. 49, no. 11, pp. 710-715, 2015.

[6] L. Kwak, S. Kremers, A. Walsh, and H. Brug, "How is your walking group running?" Health Education, vol. 106, no. 1, pp. 21-31, 2006.

[7] P. Yang, S. Dai, H. Xu, and P. Ju, "Perceived environmental, individual and social factors of long-distance collective walking in cities," International journal of environmental research and public health, vol. 15, no. 11, p. 2458, 2018.

[8] S. E. Hardy, Y. Kang, S. A. Studenski, and H. B. Degenholtz, "Ability to walk $1 / 4$ mile predicts subsequent disability, mortality, and health care costs," Journal of general internal medicine, vol. 26, no. 2, pp. 130-135, 2011.

[9] B. McKenzie, "Modes less traveled: Bicycling and walking to work in the united states, 2008-2012," US Department of Commerce, Economics and Statistics Administration, US , Tech. Rep., 2014.

[10] F. Mueller, S. O'Brien, and A. Thorogood, "Jogging over a distance: Supporting a jogging together experience although being apart," in Proc. ACM Int. Conf. on Human Factors in Computing Systems. ACM, 2007, pp. 1989-1994.

[11] F. Menzer, A. Brooks, P. Halje, C. Faller, M. Vetterli, and O. Blanke, "Feeling in control of your footsteps: conscious gait monitoring and the auditory consequences of footsteps," Cognitive neuroscience, vol. 1, no. 3, pp. 184-192, 2010.

[12] H. Murata, Y. Bouzarte, J. Kanebako, and K. Minamizawa, "Walk-in music: Walking experience with synchronized music and its effect of pseudo-gravity," in ACM Symposium on User Interface Software and Technology. ACM, 2017, pp. 177-179.

[13] E.-L. Sallnäs, "Haptic feedback increases perceived social presence," in Proc. Int. Conf. on Human Haptic Sensing and Touch Enabled Computer Applications. Springer, 2010, pp. 178-185.

[14] S. J. Lederman and R. L. Klatzky, "Haptic perception: A tutorial," Attention, Perception, and Psychophysics, vol. 71, no. 7, pp. 1439-1459, 2009.

[15] C. Pacchierotti, S. Sinclair, M. Solazzi, A. Frisoli, V. Hayward, and D. Prattichizzo, "Wearable haptic systems for the fingertip and the hand: taxonomy, review, and perspectives," IEEE Trans. Haptics, vol. 10, no. 4, pp. 580-600, 2017.

[16] J. B. F. V. Erp, H. A. H. C. V. Veen, C. Jansen, and T. Dobbins, "Waypoint navigation with a vibrotactile waist belt," ACM Trans. on Applied Perception, vol. 2, no. 2, pp. 106-117, 2005.

[17] R. W. Lindeman, J. L. Sibert, E. Mendez-Mendez, S. Patil, and D. Phifer, "Effectiveness of directional vibrotactile cuing on a building-clearing task," in Proc. ACM Int. Conf. on Human Factors in Computing Systems. ACM, 2005, p. 271. 
[18] A. Cosgun, E. A. Sisbot, and H. I. Christensen, "Guidance for human navigation using a vibro-Tactile belt interface and robot-like motion planning," in Proc. IEEE Int. Conf. on Robotics and Automation. IEEE, 2014, pp. 6350-6355.

[19] M. Aggravi, S. Scheggi, and D. Prattichizzo, "Evaluation of a predictive approach in steering the human locomotion via haptic feedback," in Proc. IEEE/RSJ Int. Conf. on Intelligent Robots and Systems, 2015, pp. 597-602.

[20] T. Lisini Baldi, S. Scheggi, M. Aggravi, and D. Prattichizzo, "Haptic Guidance in Dynamic Environments Using Optimal Reciprocal Collision Avoidance," IEEE Robot. Autom. Lett., vol. 3, no. 1, pp. 265-272, 2018.

[21] S. Scheggi, M. Aggravi, F. Morbidi, and D. Prattichizzo, "Cooperative human-robot haptic navigation," in Proc. IEEE Int. Conf. on Robotics and Automation. IEEE, 2014, pp. 2693-2698.

[22] G. Paolocci, T. Lisini Baldi, and D. Prattichizzo, "Human rendezvous via haptic suggestion," in Haptic Interaction, H. Kajimoto, D. Lee, S.-Y. Kim, M. Konyo, and K.-U. Kyung, Eds. Singapore: Springer Singapore, 2019, pp. 262-267.

[23] T. Hirano1, J. Kanebako, Y. Saraiji, R. Lalintha Peiris, and K. Minamizawa, "Synchronized running: Running support system for guide runners by haptic sharing in blind marathon," in Proc. IEEE World Haptics Conference, 2019, pp. 25-30.

[24] F. Gemperle, T. Hirsch, A. Goode, J. Pearce, D. Siewiorek, and A. Smailigic, "Wearable vibro-tactile display," 2003.

[25] I. Karuei, K. E. MacLean, Z. Foley-Fisher, R. MacKenzie, S. Koch, and M. El-Zohairy, "Detecting vibrations across the body in mobile contexts," in Proc. ACM Int. Conf. on Human Factors in Computing Systems, 2011, p. 3267.

[26] T. Lisini Baldi, G. Paolocci, and D. Prattichizzo, "Human guidance: Suggesting walking pace under manual and cognitive load," in Proc. Int. Conf. on Human Haptic Sensing and Touch Enabled Computer Applications. Springer, 2018, pp. 416-427.

[27] S. Scheggi, M. Aggravi, and D. Prattichizzo, "Cooperative Navigation for Mixed Human-Robot Teams Using Haptic Feedback," IEEE Trans. Human-Mach. Syst., vol. 47, no. 4, pp. 462-473, 2017.

[28] A. Riener, Sensor-Actuator Supported Implicit Interaction in Driver Assistance Systems, S. H. Et al., Ed. Springer, 2011, vol. 10.

[29] Apple Computer. Questions, Nike + iPod Frequently Asked. [Online]. Available: https://support.apple.com/

[30] M. Philippson, L'autonomie et la centrilasation dans le système nerveux des animaux. Falk, 1905, vol. 7.

[31] F. Delcomyn, "Neural basis of rhythmic behavior in animals," Science, vol. 210, no. 4469, pp. 492-498, 1980.

[32] Y. Miyake and T. Miyagawa, "Internal observation and co-generative interface," in Proc. IEEE Int. Conf. on Systems, Man, and Cybernetics, vol. 1. IEEE, 1999, pp. 229-237.

[33] D. Wang, M. Xu, Y. Zhanq, and J. Xiao, "Preliminary study on hapticstimulation based brainwave entrainment," in Proc. IEEE World Haptics Conference. IEEE, 2013, pp. 565-570.

[34] J. B. Dingwell and J. P. Cusumano, "Nonlinear time series analysis of normal and pathological human walking," Chaos: An Interdisciplinary Journal of Nonlinear Science, vol. 10, no. 4, pp. 848-863, 2000.

[35] H. B. Menz, S. R. Lord, and R. C. Fitzpatrick, "Acceleration patterns of the head and pelvis when walking on level and irregular surfaces," Gait \& posture, vol. 18, no. 1, pp. 35-46, 2003.

[36] E.-L. Sallnäs, "The effect of modality on social presence, presence and performance in collaborative virtual environments," $\mathrm{Ph}$.D. dissertation, KTH, 2004.

[37] T. S. Saponas, J. Lester, C. Hartung, and T. Kohno, "Devices That Tell On You : The Nike + iPod Sport Kit," Tech. Rep., 2006.

[38] D. J. Acosta and G. King, "Validating the Nike+ Wireless Sport Kit for estimating pace, distance, and energy expenditure during treadmill walking and running," vol. M.S., 2011. [Online]. Available: http://libservy.nie.edu.sg/login.php/?url=http: //search.proquest.com/docview/892713392? accountid $=28158$

[39] N. A. Kane, M. C. Simmons, D. John, D. L. Thompson, and D. R. Basset, "Validity of the Nike+ device during walking and running," International Journal of Sports Medicine, vol. 31, no. 2, pp. 101-105, 2010.

[40] Dmitry Grinberg. Nike+iPod reverse engineering. [Online]. Available: http://dmitry.gr/?r=05.Projects\&proj=05.\%20Nike\%20plus\%20iPod 